\title{
LA ATENUACIÓN LINGÜÍSTICA EN EL CORPUS PRESEEA DE SANTIAGO DE CHILE
}

Linguistic mitigation in the PRESEEA corpus of Santiago de Chile

\author{
SILVANA GUERRERO GONZÁLEZ \\ Universidad de Chile (Chile) \\ siguerrero@u.uchile.cl
}

\section{Resumen}

En este trabajo se presentan los principales resultados del estudio del fenómeno de la atenuación desde la perspectiva sociopragmática en el corpus PRESEEA de Santiago de Chile. Para cumplir este objetivo, se sigue la metodología de análisis propuesta en Cestero y Albelda (2012), Briz y Albelda (2013), Cestero y Rodríguez Alfano (2014) y Albelda et al. (2014), entre otros. De esta manera, se integran tres tipos de factores: lingüísticos, pragmático-discursivos y sociales. A partir de la revisión de nueve horas de grabación de la variedad chilena del español, registradas en 18 entrevistas semiestructuradas, se concluye que los hablantes presentan 750 actos de habla atenuados con 1731 recursos atenuadores. Se comprueba, asimismo, que la autoprotección es la principal función que cumplen los recursos de atenuación en el español chileno y que suele emplearse más la atenuación del modus, sobre todo mediante justificaciones y excusas. Finalmente, se concluye que la mayor variabilidad se produce en relación con los factores edad y nivel de instrucción de los informantes.

Palabras clave: Variación sociopragmática; atenuación; autoprotección; imagen; PRESEEA.

Abstract

This paper presents the main results of a sociopragmatic study of mitigation in the PRESEEA corpus of Santiago de Chile. The method of analysis follows the proposals of, among others, Cestero and Albelda (2012), Briz and Albelda (2013), Cestero y Rodríguez Alfano (2014) and Albelda et al. (2014), which allows to integrate the linguistic, pragmatic-discursive and social factors. From the examination of nine hours of recordings of the Chilean variety of Spanish, obtained from 18 semi-structured interviews, it is concluded that speakers produce 750 mitigated speech acts and a total of 1731 mitigating devices. It is also shown that the main function of mitigating devices in Chilean Spanish is self-protection, and that modus mitigation tends to be the most widely used type of mitigation, mainly through reasons and excuses. Finally, it is concluded that the greater variability occurs in relation to the age and level of education of participants.

Key words: Sociopragmatic variation; mitigation; self-protection; image; PRESEEA. 


\section{INTRODUCCIÓN}

En este trabajo se parte de la base de que la atenuación (o mitigación ${ }^{1}$ ) constituye un recurso retórico-comunicativo, originado por necesidades de imagen, que tiene como función mitigar o minimizar la intensidad de lo expresado y reducir lo que se calcula por los hablantes como amenazante o como menos exitoso en un determinado contexto, para los fines de la comunicación (Briz y Albelda, 2013). Esta estrategia reduce la fuerza ilocutiva del acto de habla o minimiza el contenido proposicional, de manera que permite expresar un menor compromiso con lo dicho y lograr, de manera más eficaz, las metas conversacionales de los hablantes (Albelda, 2016, 2018). Considérese el siguiente caso:

(1) hace mal / porque tengo como problemas de la memoria a corto plazo gracias a fumar tanto rato marihuana / hay una droga nueva que salió que quiero puro probarla / dicen que es así como cuatro veces el efecto de la marihuana (SCHI_M11_007) $)^{2}$.

En el ejemplo citado se está en presencia de una forma de atenuar mediante una impersonalización "dicen que", que apela al juicio de la mayoría o a un interlocutor general por medio de una forma verbal impersonal. Consiguientemente, en esta investigación se describe el fenómeno de la atenuación en el corpus del Proyecto para el Estudio Sociolingüístico del Español de España y de América (PRESEEA) de Santiago de Chile en tres dimensiones: lingüística, pragmático-discursiva y social. De esta manera, se pretende aportar resultados que posibiliten la comparación de patrones de convergencia y divergencia con otras variedades del español en el marco del PRESEEA.

En el ámbito hispánico existen múltiples trabajos de la atenuación linguiística. Por mencionar algunos de los más relevantes, se puede citar a Molina Martos (2005, 2015), Briz (2007, 2012), Briz y Estellés (2010), Albelda y Cestero (2011), Cestero (2011, 2012a, 2012b, 2014, 2015, 2017), Cestero y Albelda (2012), Briz y Albelda (2013), Samper (2013, 2017, 2018), Molina (2015), Torres y Rodríguez (2016), Albelda y Mihatsch (2017) y Albelda (2011, 2013, 2016, 2018). Para el caso del español de Chile se cuenta con las investigaciones de Puga (1997), González Riffo y Guerrero González

\footnotetext{
${ }^{1}$ En esta investigación se entiende atenuación como equivalente a mitigación. Para una revisión detallada del empleo de estos conceptos, se sugiere ver Albelda y Cestero (2011) y Albelda (2018).

${ }^{2}$ Todos los ejemplos son parte del corpus analizado. Se transcriben de manera ortográfica y al final de cada uno se codifica, entre paréntesis, la información relativa al informante. A este respecto, SCHI = Santiago de Chile; $\mathrm{H}=$ Hombre y $\mathrm{M}=$ Mujer. El primer número, luego del sexo del informante, corresponde al grupo etario: $1=$ 20-34 años; 2 = 35-54 años y $3=55$ años y más. El segundo número corresponde al nivel educacional del informante: 1 = estudios básicos completos o incompletos; 2 = estudios medios completos o incompletos y $3=$ estudios universitarios completos o incompletos. El número que sigue al guion corresponde al número correlativo que reciben los sujetos que conforman la muestra. $\mathrm{E}=$ entrevistador e I = informante. Solo se analizan los actos de habla del informante.
}

54 | AlPHA No 52 (Julio 2021) PÁGS. 53-76. ISSN 07 16-4254 
(2016, 2017, 2018), González Riffo (2017), González Riffo y Guerrero González (2018) y Rodríguez Alfano (2018). Los estudios citados abordan la atenuación desde dos grandes paradigmas: el de la variación y el de los géneros discursivos.

Esta investigación, de carácter cuantitativo y cualitativo, se constituye como el primer acercamiento al análisis de las estrategias de atenuación como fenómeno variable en el habla de Chile, en un corpus oral y actual de entrevistas semiestructuradas, adscritas al corpus del PRESEEA. Se trata de un estudio acerca de la atenuación que intenta contribuir a las investigaciones que se han enfocado en fenómenos discursivos a partir de los presupuestos teóricos de la pragmática y de la sociolinguística. En consecuencia, el trabajo propuesto contribuye a los estudios en sociopragmática del español, pero más particularmente a aquellos que se centran en la variedad chilena. Asimismo, el trabajo aporta a la reflexión en torno a cómo los hablantes construyen y negocian su identidad en la interacción con base en la idea de compromiso discursivo.

\section{FUNDAMENTOS TEÓRICOS}

Este trabajo, cuyo origen es la necesidad de generar resultados que permitan comparar patrones de convergencia y divergencia con otras variedades del español en el contexto del PRESEEA, específicamente en torno al fenómeno de la atenuación, se concibe siguiendo, en lo fundamental, los presupuestos teórico-metodológicos de Cestero y Albelda (2012), Briz y Albelda (2013) y Albelda et al. (2014). En este sentido, las definiciones y fundamentos teóricos que respaldan el estudio son similares a los que se adoptan en los trabajos recién citados, aunque con algunas actualizaciones.

Debido a que la atenuación constituye una estrategia lingüística que utilizan los hablantes para distanciarse de aquello que dicen, cuyo propósito es conseguir un acercamiento social con otros sujetos (Briz, 1995; Briz y Albelda, 2013), debe estudiarse en un sentido que considere que se trata de un fenómeno que no solo varía según el contexto situacional de la interacción (Briz y Albelda, 2013), sino que también varía socialmente (Cestero y Albelda 2012).

Considerando lo anteriormente expuesto, esta investigación considera tres de las cinco variables (o factores) que propiciarían el uso de los atenuantes, según Cestero y Albelda (2012): 1) los factores lingüísticos y dentro de ellos el número de estrategias de atenuación empleadas en cada acto de habla atenuado y los recursos linguísticos de atenuación, 2) los factores pragmáticos y discursivos solo en lo referido a la función general del atenuante en el discurso y 3) los factores sociales, 
incluyendo las variables sexo, edad y nivel de instrucción. Se presenta, además, la revisión general de los tipos de atenuación ${ }^{3}$.

\subsection{TIPO DE ATENUACIÓN}

El tipo de atenuación realizada, esto es, la reducción del valor significativo de un enunciado, de lo que se dice, corresponde a atenuación del dictum o de la estructura interna del acto de habla, como ocurre, por ejemplo, mediante el empleo de diminutivos. Cuando se atenúa la fuerza ilocutiva de un acto de habla, de lo que se hace, estamos ante la atenuación del modus; es el caso de las justificaciones y de las excusas. Si se atenúa el valor significativo de un enunciado y de su fuerza ilocutiva a la vez, se trata de atenuación del dictum y del modus.

Los tipos de atenuación son la primera variable considerada en este trabajo. En el siguiente apartado se precisa, con exactitud, cómo se distribuyen los recursos de atenuación en cada uno de los tres tipos descritos.

\subsection{FACTORES LINGÜÍSTICOS: RECURSOS LINGÜÍSTICOS Y PARALINGÜÍSTICOS QUE SE UTILIZAN PARA ATENUAR}

En el estudio coordinado de la atenuación con los materiales del PRESEEA ${ }^{4}$ se han propuesto 25 tipos diferentes de recursos lingüísticos y paralingüísticos de atenuación. Algunos de los trabajos que mejor ejemplifican el análisis de estos recursos son el de Cestero y Albelda (2012), los de Cestero (2015 y 2017) y el de Albelda (2018). La taxonomía propuesta por estas autoras es la siguiente:

1. Modificación morfológica interna mediante sufijos (diminutivos).

2. Modificación morfológica externa mediante cuantificadores, minimizadores, aproximativos, difusores significativos y entrecomillado.

3. Selección léxica (lítotes y eufemismos).

4. Empleo de palabras extranjeras u onomatopeyas.

5. Modificación temporal del verbo (condicional por presente o imperativo; imperfecto por presente o imperativo; futuro por presente).

6. Expresión de aserciones en forma de duda o de probabilidad mediante el empleo de verbos o adverbios modales de pensamiento, de duda y de probabilidad.

\footnotetext{
${ }^{3}$ No se incluyen en esta primera indagación los factores geográficos y culturales, los factores lingüísticos alusivos a la posición sintáctica del atenuante en el acto de habla, los factores pragmáticos y discursivos acerca de la carga semántico-pragmática del mensaje ni del tipo de acto de habla atenuado.

${ }^{4}$ http://preseea.linguas.net/Portals/0/Gu\%C3\%ADa\%20de\%20Estudios\%20de\%20la\%20Atenuaci\%C3\%B3 n\%20en\%20corpus\%20PRESEEA.pdf

56 | AlPHA № 52 (JuLIO 2021) PÁGS. 53-76. ISSN 07 16-4254
} 
7. Expresión de aserciones en forma de duda o de probabilidad mediante indicación de incertidumbre o incompetencia, o fingimiento de ignorancia.

8. Acotación de la opinión a la propia persona o a un determinado ámbito o territorio personal, como medida cautelar que restringe la opinión.

9. Negación del supuesto de lo que se quiere pedir o preguntar.

10. Inclusión en una petición del posible rechazo del interlocutor o expresión de improbabilidad de que se conceda lo que se solicita.

11. Petición de disculpas ante una interrupción, una pregunta, un favor, etc.

12. Modificaciones del acto de habla que restringen lo dicho mediante construcciones sintácticas condicionales, concesivas o temporales, o uso de unidades fraseológicas estereotipadas.

13. Justificaciones y excusas.

14. Utilización o simulación de estilo directo para expresar las propias palabras o la propia opinión.

15. Elipsis de la conclusión (estructuras suspendidas).

16. Uso de fórmulas fáticas de petición de consentimiento.

17. Impersonalizaciones que apelan al juicio de la mayoría o a un interlocutor general mediante pronombres, formas verbales impersonales u otras formas de despersonalización del origen deíctico del enunciado.

18. Impersonalizaciones con expresiones que evitan la aseveración por parte del hablante y recurren a una causa o presión externa que motiva lo dicho.

19. Impersonalizaciones mediante marcadores discursivos que inciden en la franqueza de lo dicho y lo objetivan.

20. Empleo de marcadores del discurso atenuantes correctores para minimizar la disconformidad dialógica o para rebajar y proteger la propia imagen monológicamente.

21. Uso de marcadores del discurso que indican consecuencia lógica.

22. Movimientos concesivos para minimizar la disconformidad dialógica o para atenuar y proteger la propia imagen monológicamente.

23. Comparación como forma de justificación.

24. Reducción del formato del acto de habla.

25. Utilización de elementos paralingüísticos, como la risa y las vacilaciones.

Los cuatro primeros recursos corresponden a la atenuación del dictum. Los recursos que van del 5 al 25 constituyen estrategias de atenuación del modus. Cuando se combinan recursos del 1 al 4 y del 5 al 25 se considera atenuación del dictum y del modus de manera conjunta (cf. Cestero y Albelda (2012), Briz y Albelda (2013), Cestero y Rodríguez Alfano (2014), Cestero (2014) y Albelda et al. (2014)). 


\subsection{FACTORES PRAGMÁTICOS Y DISCURSIVOS: FUNCIÓN GENERAL DEL ATENUANTE EN EL DISCURSO}

Tal como sugieren Cestero y Albelda (2012), en relación con los aspectos pragmáticos y discursivos que afectan el uso de los recursos atenuantes, en esta investigación, primeramente se analizaron siete propósitos o fines con los que se atenúa en el corpus en estudio: reducir la repercusión de una generalización o evidencia o el efecto de lo dicho; evitar o reducir el compromiso del hablante con lo dicho; realizar actividades de autoimagen; manifestar o buscar acuerdo; reparar o mitigar una amenaza a la imagen del oyente; evitar imponer el yo, y justificar el desacuerdo o evitar un posible desacuerdo.

Luego, estos fines se reagrupan, tal como se sugieren las citadas autoras, en tres funciones básicas de la atenuación, que son, finalmente, de las que se da cuenta en este trabajo: 1) autoprotección, esto es, el uso de atenuadores para evitar o reducir el compromiso del hablante con lo dicho o su repercusión y para salvar la imagen propia; 2) prevención, en cuyo caso se tiende a evitar las repercusiones de lo dicho o hecho e imponer la opinión propia, buscando acuerdo o justificando el desacuerdo; y, 3) reparación, cuya función es mitigar o reparar una amenaza al interlocutor.

\subsection{FACTORES SOCIALES: CARACTERÍSTICAS SOCIALES DE LOS HABLANTES}

Respecto de los factores sociales, y siguiendo los presupuestos metodológicos del proyecto PRESEEA, se trabaja con el sexo de los informantes (mujer y hombre), la edad (jóvenes o primer grupo etario -de 20 a 34 años-, adultos o segundo grupo etario - de 35 a 54 años- y tercer grupo de edad -55 años o más-), y el nivel de instrucción (estudios básicos -primarios-, estudios secundarios -medios- y estudios superiores - universitarios-).

\section{METODOLOGÍA}

\subsection{EL CORPUS Y LOS INFORMANTES}

El corpus que sirvió de base para esta investigación corresponde a 18 entrevistas semidirigidas del corpus del PRESEEA de Santiago de Chile. De cada entrevista se seleccionaron 30 minutos de grabación (minutos 10 al 40), lo que, en la práctica, constituye 9 horas de registro de discurso natural (o vernáculo).

Los informantes son representativos de la comunidad de habla santiaguina y están estratificados por sexo, edad y nivel de instrucción, como se describe a continuación: se trata de una muestra uniforme, que incluye las variables sociales antes mencionadas. En la práctica, son 9 mujeres y 9 hombres, divididos en 6 hablantes por grupo de edad, según los criterios de PRESEEA, 20-34, 35-54 y 55 años y más. Finalmente, estos hablantes 
representan a los tres niveles de instrucción que se contemplan en el proyecto: 6 pertenecen al nivel de estudios alto, 6 al medio y 6 al bajo.

\subsection{PROCEDIMIENTOS ANALÍTICOS}

Para llevar a cabo el estudio de la atenuación, se utilizó como unidad de análisis el acto de habla y se adoptó la metodología de análisis propuesta en Cestero y Albelda (2012), Briz y Albelda (2013), Cestero y Rodríguez Alfano (2014), Cestero (2014) y Albelda et al. (2014). En consecuencia, se siguieron cuatro pasos fundamentales:

i. En primera instancia, se considera el estudio del tipo de atenuación realizada.

ii. En la dimensión lingüística, se revisan los recursos lingüísticos que se utilizan para atenuar.

iii. A nivel pragmático-discursivo se da cuenta de las funciones básicas de la atenuación: autoprotección, prevención y reparación.

iv. En relación con la función social, se presenta el análisis de los recursos de atenuación según los factores sexo, edad y nivel de instrucción de los hablantes.

Se realizó, primero, un análisis inductivo y, luego, se completaron los hallazgos con estadística descriptiva. Esta decisión se justifica porque se siguen los supuestos teórico-metodológicos de la sociopragmática.

\section{PRESENTACIÓN Y DISCUSIÓN DE LOS RESULTADOS}

En el análisis del corpus se registraron 750/1901 (39,5\%) actos de habla atenuados con 1731 recursos atenuadores. Más concretamente, se puede precisar que, cuando se atenúa, en la variedad de Santiago de Chile suelen utilizarse entre uno y dos recursos destinados a mitigar, por cada acto de habla. De los 750 actos de habla registrados, en 325 se emplea un solo recurso cuya función es atenuar (43,3\%) y en 192 actos de habla se usan dos recursos atenuadores $(25,6 \%)$. Por el contrario, se registraron unos pocos casos (19) en los que se emplean entre 10 y 15 atenuantes por cada acto de habla. En un rango intermedio, esto es, en 95 actos de habla se emplearon 3 recursos atenuantes (12,7\%); en 54 actos se incluyeron 4 atenuantes (7,2\%); en 32 actos de habla se usaron 5 recursos atenuadores $(4,2 \%)$; en 16 casos se usaron 6 atenuantes $(2,1 \%)$; en 9 actos de habla se incluyeron 7 recursos atenuadores $(1,2 \%)$; y, en 8 actos de habla se usaron 4 atenuadores de manera conjunta $(1 \%)$.

Un segundo hallazgo relevante es que en el corpus PRESEEA de Santiago de Chile se observó que suele emplearse más la atenuación del modus (497 actos de habla atenuados), seguida de la atenuación del dictum y el modus (108 casos) y que, en tercer lugar, se ubica la frecuencia de los casos de atenuación por medio del dictum (145 actos de habla). Este hallazgo hay que observarlo considerando que de los 25 recursos 
analizados, solo cuatro son del dictum y, de ellos, se registraron solo tres en el corpus. En cualquier caso, este patrón de empleo coincide con el registrado por Cestero (2012a) para el habla de Madrid, con el de Cestero y Albelda (2012) para Madrid y Valencia, y con el de Samper (2013) para Las Palmas de Gran Canaria.

La atenuación del modus se ejemplifica en casos como:

(2) lo que a uno le gusta son los sentimientos / uno parte por ahí (SCHI_H21_013).

En este caso se puede observar un acto de habla asertivo, donde se atenúa la opinión del hablante mediante una impersonalización. Se reduce así la fuerza ilocutiva de un acto de habla y, con ello, la actitud del hablante al emitir el acto de habla, debido a que no se impone ante su interlocutor. Por tanto, se minimiza también la intención detrás del acto de habla. Se podría argüir que es un acto expresivo (declaración de afecto) -aunque indirecto-, pero la impersonalización lo "asertiviza". También se podría señalar que el empleo de "uno" se condice con la idea de querer decir que lo que uno piensa no es solo de uno, sino que otros también lo piensan y, en consecuencia, no es algo subjetivo sino más bien generalizado. Respecto del empleo de "uno" asimismo González y Hugo (2012) precisan que, además del carácter deíctico, tiene usos considerados como un "encubrimiento del yo" (las comillas son de los autores); se trataría de una especie de subterfugio linguístico para que alguien hable de sí mismo. Este aspecto coincide con lo que se plantea desde la función atenuadora de "uno".

La atenuación del dictum y el modus se refleja en el ejemplo (3):

(3) he vivido en todos lados / en las partes buenas / las partes más o menos / y en las partes buenas / cachái / pero / no sé / encuentro todo igual / no encuentro la gran diferencia / solamente que / se / encuentro extraño que pasen cosas malas huevón en las partes buenas / cachái / pero eso / si uno mientras que no / no mire a lo aje </palabra cortada>/ lo ajeno/ estábien (SCHI_H11_001).

El ejemplo (3) consiste en una expresión de una aserción (opinión) donde se combina la modificación morfológica externa por medio del uso de "solamente" con la impersonalización de aquello que se va a señalar ("uno"). El hablante generaliza para convencer al interlocutor de que el barrio en el que vive es tranquilo, porque no hay robos.

Por último, se presenta atenuación del dictum en actos de habla como:

(4) si y después la moto / la moto quedó en la casa pues / y casi nadie creía como habíamos / pensaban que nosotros nos habíamos matado también (SCHI_H22_049). 
En este caso se utiliza una modificación morfológica externa; suelen utilizarse aproximativos o difusores del significado, nuevamente para autoproteger la imagen propia. Con el empleo de "casi" en el acto de habla (del mismo que en "solamente" en el ejemplo (3)), se reduce el valor significativo del enunciado o de lo que se dice, ya que lo que se modifica es la estructura interna del acto de habla.

\subsection{FACTORES LINGÜÍSTICOS: RECURSOS LINGÜÍSTICOS Y PARALINGÜÍSTICOS ATENUANTES}

En la dimensión lingüística, en Santiago de Chile se observó el empleo de 21 de las 25 categorías de recursos de atenuación propuestas en la ficha elaborada por Cestero y Albelda (2012). No se registraron atenuadores correspondientes a selección léxica, recursos para negar el supuesto de pedir o preguntar, inclusión en una petición del posible rechazo del interlocutor o expresión de improbabilidad ni elementos destinados a rebajar el acto de habla.

Respecto de los recursos de atenuación utilizados en el corpus de habla del PRESEEA de Santiago de Chile, los atenuadores empleados de manera más frecuente son los que se presentan en la tabla que sigue ${ }^{5}$.

Tabla 1. Recursos de atenuación más frecuentes en el corpus PRESEEA-Santiago

\begin{tabular}{|l|c|c|l|}
\hline \multicolumn{1}{|c|}{ Recurso } & Frecuencia & $\%$ & \multicolumn{1}{|c|}{ Ejemplo } \\
\hline Justificaciones & $304 / 1731$ & 17,6 & $\begin{array}{l}\text { (5) me han penado varias veces / es } \\
\text { que igual soy perceptible de / yo } \\
\text { pues / yo / siento ruidos / eso } \\
\text { (SCHI_H11_001). }\end{array}$ \\
\hline $\begin{array}{l}\text { Marcadores discursivos } \\
\text { correctores }\end{array}$ & $256 / 1731$ & 14,8 & $\begin{array}{l}\text { (6) yo estuve } \boldsymbol{a} \text { ver yo / cuando era } \\
\text { más / más niña (SCHI_M12_043). }\end{array}$ \\
\hline $\begin{array}{l}\text { Recursos de expresión de } \\
\text { duda mediante verbos o } \\
\text { adverbios }\end{array}$ & $163 / 1731$ & 9,4 & $\begin{array}{l}\text { (7) lo tengo todo armado pero estoy } \\
\text { muy muy dejado /y el gimnasio si lo } \\
\text { ha participé en gimnasio me gustó / } \\
\text { pero no sécreo que la gente que vaal } \\
\text { gimnasio tiene cierta características } \\
\text { especiales (SCHI_H23_085). }\end{array}$ \\
\hline
\end{tabular}

\footnotetext{
${ }^{5}$ En las Tablas 1, 4, 5 y 6 se incluyen solo las categorías en las que se cuantificaron 100 casos o más. Se consideró este número de casos como de "alta frecuencia".
} 


\begin{tabular}{|l|l|l|l|}
\hline $\begin{array}{l}\text { Recursos de expresión de } \\
\text { duda mediante indicación de } \\
\text { incertidumbre }\end{array}$ & $148 / 1731$ & 8,5 & $\begin{array}{l}\text { (8) no sé / debo saber tenido treinta } \\
\text { y tantos (SCHIM33_103). }\end{array}$ \\
\hline Movimientos concesivos & $144 / 1731$ & 8,5 & $\begin{array}{l}\text { (9) sí pero / el frío / sí igual hubo } \\
\text { harto frío / sí igual hizo harto frío } \\
\text { (SCHI_H12_037). }\end{array}$ \\
\hline $\begin{array}{l}\text { Impersonalizaciones que } \\
\text { apelan al juicio de la } \\
\text { mayoría }\end{array}$ & $129 / 1731$ & 7,5 & $\begin{array}{l}\text { (10) ahora se dice más joven / pero } \\
\text { de verdad / uuh la persona decía / } \\
\text { no te casas a lo después de los } \\
\text { veinte (SCHI_M22_055). }\end{array}$ \\
\hline $\begin{array}{l}\text { Recursos de modificación } \\
\text { morfológica externa }\end{array}$ & $128 / 1731$ & 7,4 & $\begin{array}{l}\text { (11) I: las dos cosas / yo creo que } \\
\text { van a entablar relaciones/ } \\
\text { conocer gente y también a cuidar } \\
\text { de su estado físico y cuidar de la } \\
\text { salud porque el ejercicio hace } \\
\text { bien// E: _ha conocido algún caso } \\
\text { en que se confirme esta afirmación } \\
\text { que nos pueda relatar? // I: no no / } \\
\text { pero no solamente lo que veo en la } \\
\text { tele va mucha gente / los modelos } \\
\text { creo que van para conocerse }\end{array}$ \\
$\begin{array}{l}\text { (SCHI_H21_013). } \\
\text { Marcadores discursivos de } \\
\text { consecución lógica }\end{array}$ & $123 / 1731$ & $7,1 \%$ & $\begin{array}{l}\text { veintinueve años que ya estaba la } \\
\text { tele en Estados Unidos / entonces } \\
\text { los norteamericano le llevan } \\
\text { veintinueve años de ventaja al } \\
\text { sudamericano (SCHI_H32_061). }\end{array}$ \\
\hline
\end{tabular}

Estos datos son similares en cuanto a estrategias comunicativas a los de Cestero y Albelda (2012) para el habla de Madrid y Valencia, aunque para el caso específico de Madrid (Cestero, 2012a) es el paralenguaje el que destaca por su alta frecuencia de uso. Un análisis contrastivo relevante en Madrid, Valencia, Las Palmas y Santiago es el que se halla en Albelda et al. (2020). También se observan similitudes con el español de Barranquilla (Reyes y Rodríguez, 2016), donde el “como", el "no saber" y las expresiones de duda registran frecuencias de empleo altas. 
Con frecuencias que van entre 76 y un caso se ubican los recursos para acotar la opinión propia (76 casos), por ejemplo mediante el atenuante "yo digo que"; el paralenguaje (63 casos), reflejado en el empleo de risas y alargamientos sobre todo para reparar; los recursos de modificación morfológica interna (61 casos), donde los sufijos -ito e ita- son los que permiten atenuar el contenido de lo dicho; el estilo directo (55 casos), en cuyo caso se usa esta forma de discurso referido para no dar la opinión propia y no comprometerse con lo dicho; las fórmulas fáticas (39 casos), como el “¿cachái?" equivalente a "¿entiendes?”; la comparación (12 casos), que permite generalizar ciertas opiniones, como ocurre con el uso de "todo el mundo"; la impersonalización mediante marcadores discursivos que inciden en la franqueza de lo dicho y lo objetivan (9 casos), donde destaca el uso de "la verdad (es que)"; la modificación temporal del verbo (7 casos), como en "diría"; la elipsis de la conclusión (5 casos), la petición de disculpas y la impersonalización con expresiones que evitan la aseveración por parte del hablante y recurren a una causa o presión externa que motiva lo dicho ( 3 casos); la modificación del acto de habla ( 2 casos) con expresiones como "si no me equivoco"; $y$, por último, los extranjerismos y onomatopeyas (1 caso).

Se presenta a continuación el gráfico 1, que ilustra, mediante porcentajes, los datos proporcionados.

Gráfico 1. Recursos de atenuación en el corpus PRESEEA-Santiago

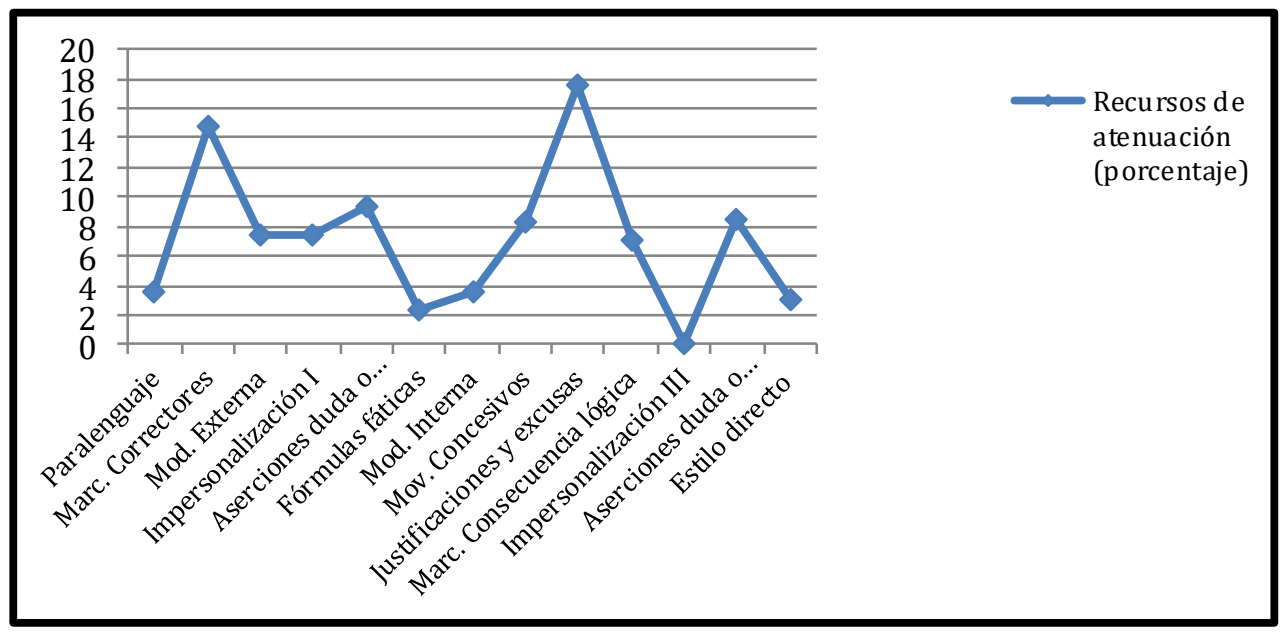




\subsection{FACTORES PRAGMÁTICO-DISCURSIVOS: FUNCIONES BÁSICAS DE LA ATENUACIÓN}

En lo que respecta a las funciones básicas de la atenuación, se puede apreciar que en Santiago de Chile la autoprotección es la principal función que cumplen los recursos de atenuación; en concreto, esta función se encuentra en el 85,6\% (648/750) del total de actos de habla. La prevención se utiliza en el 12,9\% (96/750) de los actos y la reparación solo en el 1,5\% (6/750) del total de casos. En el español de Valencia (Albelda, 2018) se observa un patrón de comportamiento casi idéntico.

El ejemplo (12) ilustra claramente la función de autoprotección:

(12) me he estado desilusionando de ese de ese tipo de cine de terror / porque han salido películas muy malas / ya / no sé / es como difícil ver // me gustan más las películas / han salido mejores películas de suspenso / de drama / yo creo que más por ese lado me me estoy yendo ahora (SCHI_M12_043).

En el ejemplo citado, la informante está argumentando de su posición respecto del cine actual. En la cita se aprecia el empleo de tres diferentes recursos de atenuación destinados a autoproteger su imagen, toda vez que su opinión constituye una crítica. En este sentido, evita el compromiso con lo dicho, en primer lugar, expresando una duda mediante una forma de incertidumbre "no sé", pese a que ya ha explicitado que las películas que han salido son "muy malas". Luego, en lugar de señalar directamente que es difícil ver buen cine de terror, justifica su respuesta con "es como". Finalmente, utiliza un verbo que le permite expresar duda "creo", pero acotando la opinión a su propia persona "yo creo", de manera que quede claro que su opinión no es definitiva.

En el ejemplo (13) se observa uno de los pocos casos de prevención registrados en la muestra. Debe recordarse que la estrategia de prevención es la que busca el acuerdo o justificar el desacuerdo.

(13) E: pero ¿tú encuentras que eso es bueno / o sea / todas los las diferencias son buenas? / I: buenísimas // E: pero hay cosas negativas / lo que tú estás diciendo de los vicios // I: eso sí pues // E: ¿eso es malo? // I: requete re contra malo / porque un cabro 6 / el cabro en vez de beneficiarse va en retroceso / porque el niño que fuma marihuana / o bebe o fuma / tú tienes que partir de la base que el cuerpo humano no se hizo para eso / y si está fumando marihuana / se está afectando directamente las neuronas pues / y le está fallando los / ligerito el cabro ya empieza a a a ya no ya no no piensa / balbucea (SCHI_H31_061).

\footnotetext{
${ }^{6}$ En español de Chile, niño, joven.

64 | AlPHA No 52 (Julio 2021) PÁGS. 53-76. ISSN 07 16-4254
} 
En (13), el hablante proporciona su opinión de lo negativo que es consumir marihuana, sobre todo, en los adolescentes. En este caso, en lugar de autoproteger su imagen, atenúa su discurso en la dirección de buscar el acuerdo de su interlocutor. Para ello, elabora un discurso donde, mediante el uso de una justificación "porque", intenta convencer al interlocutor de su postura, proporcionando evidencias de por qué el consumo de marihuana es negativo. Sumado a esto, se halla el uso de "requeté re contra", que permite intensificar para conseguir el acuerdo con el interlocutor, por tanto, también es una estrategia de imagen. La intensificación como mecanismo de atenuación ya tiene algunos estudios empíricos (cf. Albelda y Nihatsch, 2017).

La reparación, como ya se ha precisado, casi no aparece en la muestra analizada. Se proporciona igualmente el ejemplo (14), cuyo contexto discursivo permite comprender cómo el hablante mitiga o repara una amenaza.

(14) I: es que / es penca ${ }^{7}$ / lo encuentro penca / porque antes cuando / para ir al / por ejemplo ir a / a la Estación Central / yo tenía que tomar una pura locomoción de aquí a mi casa / ahora tengo que hacer como tres / tomar tres huevadas para llegar allá a la estación pues // E: sí pues // I: gasto más / o sea / bueno no es tanto lo que gasto pero es que igual pues (SCHI_M21_019).

En el ejemplo citado funcionan las estrategias de atenuación de manera paralela. Inicialmente, el hablante argumenta su postura negativa frente al sistema de Transporte de Santiago de Chile y autoprotege su imagen ("es que" y "encuentro"). Luego, para prevenir el desacuerdo de su interlocutor, justifica su respuesta ("porque") y vuelve a mitigar con el empleo de "como" (se opone "como tres" a "una pura") y, finalmente, repara lo dicho autocorrigiéndose, porque primero señala que gasta más [dinero], pero se autocorrige al incluir atenuadores que constituyen marcadores discursivos correctores ("o sea" y "bueno"), sumados al uso de la justificación con "es que". El uso de marcadores no en todos los casos tiene la función de reparar. Así, es relevante considerar siempre el contexto de enunciación, para determinar frente a qué estrategia de atenuación estamos. Hay que considerar, asimismo, el tipo de género analizado, ya que las funciones podrían ser menos transparentes en la conversación coloquial.

\subsection{FACTORES SOCIALES}

\subsubsection{RECURSOS DE ATENUACIÓN Y FACTORES SOCIALES}

En lo que respecta a la variedad de Santiago de Chile, el análisis de los datos indica que los hombres atenúan más que las mujeres, aunque se trata de frecuencias de empleo casi idénticas: $876(50,6 \%)$ casos de recursos atenuadores en los actos de habla de

\footnotetext{
${ }^{7}$ En español de Chile, adjetivo que indica valoración acerca de la mala calidad de algo o alguien.
} 
hombres y 855 (49,4\%) en los de mujeres. Ocurre lo mismo en el habla de Las Palmas de Gran Canaria (Samper, 2013), pero las diferencias se acentúan en el habla de Madrid y de Valencia (Cestero y Albelda, 2012)

En relación con el factor edad, se observa que el empleo de recursos atenuadores disminuye conforme aumenta la edad: 732 (42,3\%) casos en los hablantes jóvenes, 644 $(37,2 \%)$ casos en los hablantes del grupo de edad intermedia y 355 (20,5\%) casos en los hablantes del tercer grupo de edad. Este resultado es similar al de Samper (2013), pero difiere de los de Cestero y Albelda (2012). Los resultados en contraste pueden constatarse de manera más evidente en Albelda et al. (2020).

Respecto de la variable nivel de instrucción, en esta comunidad de habla, los hallazgos indican que son los informantes con nivel de instrucción más alto quienes menos atenúan (397 casos, 22,9\%), mientras que los hablantes de los niveles de instrucción bajo y medio presentan comportamientos similares: 602 casos $(34,2 \%)$ en el primer nivel de instrucción y $732(42,9 \%)$ en el segundo. Estos resultados son inversos a los de Las Palmas de Gran Canaria (Samper, 2017).

Tabla 2. Atenuación en el corpus PRESEEA-Santiago de Chile: sexo, edad y nivel de instrucción

\begin{tabular}{|c|c|c|c|c|c|c|}
\hline \multirow{2}{*}{\multicolumn{3}{|c|}{ Nivel de instrucción }} & \multicolumn{3}{|c|}{ Edad } & \multirow{3}{*}{\begin{tabular}{|c|} 
Totales \\
253 \\
\end{tabular}} \\
\hline & & & \multirow{2}{*}{$\begin{array}{c}\begin{array}{c}\mathbf{2 0 - 3 4} \\
\text { años }\end{array} \\
107\end{array}$} & \multirow{2}{*}{$\begin{array}{c}\begin{array}{c}35-55 \\
\text { años }\end{array} \\
92\end{array}$} & \multirow{2}{*}{$\begin{array}{c}\begin{array}{c}\mathbf{5 5 +} \\
\text { años }\end{array} \\
54\end{array}$} & \\
\hline \multirow{2}{*}{ Estudios primarios } & \multirow{2}{*}{ Sexo } & Mujer & & & & \\
\hline & & Hombre & 160 & 121 & 68 & 349 \\
\hline \multirow{2}{*}{ Estudios secundarios } & \multirow{2}{*}{ Sexo } & Mujer & 182 & 168 & 90 & 440 \\
\hline & & Hombre & 116 & 113 & 63 & 292 \\
\hline \multirow{2}{*}{ Estudios superiores } & \multirow{2}{*}{ Sexo } & Mujer & 62 & 59 & 41 & 162 \\
\hline & & Hombre & 105 & 91 & 39 & 235 \\
\hline \multicolumn{3}{|l|}{ Totales } & 732 & 644 & 355 & 1731 \\
\hline
\end{tabular}

La mayor variabilidad, como se aprecia en la Tabla 2, se produce en las mujeres, ya que las que tienen estudios universitarios -sobre todo las de 55 años y más-emplean muy pocos recursos atenuadores (162) en comparación al resto de los hablantes de la muestra, que se sitúan siempre por sobre los 235 casos, independiente del factor sexo. Por oposición a este dato, las mujeres con estudios secundarios, en especial aquellas que tienen entre 20 y 34 años, son las que utilizan recursos atenuadores con mayor frecuencia, llegando a registrar, sin considerar el factor edad, 440 casos, lo que corresponde al 25,4\% (o un cuarto) del total de la muestra. Asimismo, en el grupo de estudios primarios, las 
mujeres de edad intermedia presentan poca frecuencia de empleo de atenuantes frente a las de la misma edad, pero con estudios secundarios.

Respecto de esta variedad de español, también es interesante que la variabilidad de edad presenta una evidente disminución de empleo de recursos atenuantes entre los hablantes del tercer grupo etario, quienes registran menos de la mitad de los casos de los hablantes del grupo de edad joven. Esto se genera, de manera muy evidente, porque en todos los niveles de instrucción, los hablantes del grupo de 20 a 34 años suelen emplear más de 100 recursos atenuadores, excepto en el grupo de mujeres jóvenes con estudios superiores.

Las diferencias marcadas por acto de habla se presentan a continuación.

Tabla 3. Actos de habla con atenuación en el corpus PRESEEA-Santiago de Chile: sexo, edad y nivel de instrucción

\begin{tabular}{|c|c|c|c|c|c|c|}
\hline \multirow{2}{*}{\multicolumn{3}{|c|}{ Nivel de instrucción }} & \multicolumn{3}{|c|}{ Edad } & \multirow{3}{*}{$\begin{array}{c}\text { Totales } \\
148\end{array}$} \\
\hline & & & \multirow{2}{*}{$\begin{array}{c}\begin{array}{c}20-34 \\
\text { años }\end{array} \\
47\end{array}$} & \multirow{2}{*}{$\begin{array}{c}\begin{array}{c}35-55 \\
\text { años }\end{array} \\
62\end{array}$} & \multirow{2}{*}{$\begin{array}{c}\begin{array}{c}55+ \\
\text { años }\end{array} \\
39\end{array}$} & \\
\hline \multirow{2}{*}{ Estudios primarios } & \multirow{2}{*}{ Sexo } & Mujer & & & & \\
\hline & & Hombre & 59 & 57 & 27 & 143 \\
\hline \multirow{2}{*}{ Estudios secundarios } & \multirow{2}{*}{ Sexo } & Mujer & 48 & 45 & 44 & 137 \\
\hline & & Hombre & 52 & 40 & 30 & 122 \\
\hline \multirow{2}{*}{ Estudios superiores } & \multirow{2}{*}{ Sexo } & Mujer & 33 & 30 & 33 & 96 \\
\hline & & Hombre & 49 & 32 & 23 & 104 \\
\hline \multicolumn{3}{|l|}{ Totales } & 288 & 266 & 196 & 750 \\
\hline
\end{tabular}

Los datos según actos de habla atenuados reflejan en gran medida los expuestos en la Tabla 2. De este modo, hombres y mujeres se comportan de manera casi idéntica, aunque, en este caso, las mujeres presentan más actos de habla atenuados que los hombres (12 casos de diferencia). La edad muestra el mismo patrón descendente al avanzar en los grupos etarios y, finalmente, el nivel de instrucción también permite concluir que los actos de habla atenuados se usan menos cuando los hablantes tienen más años de escolaridad.

Junto con los datos generales, hay ciertos hallazgos más específicos que conviene comentar. Se trata de hallazgos referidos a la relación del empleo total de actos de habla atenuados en relación con las variables sociales.

Se observa variabilidad en relación con el factor sexo. Las mujeres, como ha quedado expresado en la Tabla 3, son quienes atenúan sus actos de habla con una frecuencia un poco más alta que la que registran los hombres. Ellas, en 4 actos de habla, emplean recursos atenuantes que van entre los 10 y los 12 casos, lo que, en porcentajes, representa el 2,6\% y el 3,1\% del total de actos de habla atenuados por mujeres (381). Los 
hombres, por su parte, en 6 actos de habla atenúan entre 10 y 15 oportunidades, lo que representa el 2,7\% y el $4 \%$ del total de actos atenuados por hombres (369). Sin embargo, en 174 actos las mujeres atenúan solo una vez y en 102 actos de habla emplean dos recursos atenuantes, lo que, de manera conjunta, representa el 72,4\% del total de casos. En el caso de los hombres, en 151 oportunidades atenúan una vez y en 95 ocasiones atenúan dos veces, es decir, en el 66,6\% del total de casos los hombres atenúan entre una y dos ocasiones por cada acto de habla. El resto de las frecuencias de atenuantes emitidos por mujeres, es decir, cuando atenúan entre 3 y 9 ocasiones por acto de habla emitido, constituyen $25,7 \%$ de los casos totales. Los hombres, en tanto, presentan mayor variabilidad, con el $31,7 \%$ de los usos.

En relación con el factor edad se observa que de los 288 actos de habla atenuados por hablantes del primer grupo de edad, en 105 ocasiones se atenúa una vez $(36,4 \%)$; en 77 casos se atenúa dos veces (26,7\%); en 43 oportunidades se atenúa 3 veces (14,9\%); y, en 4 ocasiones se atenúa entre 10 y 15 veces. En su conjunto, cuando se atenúa entre 4 o 9 veces por cada acto de habla, se trata de 59 casos $(20,5 \%)$ del total de actos atenuados por el grupo de edad joven. En el segundo grupo etario, del total de 266 actos de habla atenuados, en 113 casos se atenúa solo una vez (42,5\%); en 64 ocasiones se atenúa dos veces (24\%); y, en 84 actos de habla se atenúa entre tres y 9 veces $(31,6 \%)$. Además, en 3 actos de habla se atenúa entre 11 y 15 ocasiones. Por último, cuando los hablantes representan al tercer grupo de edad, atenúan 196 actos de habla, que, en la práctica, están distribuidos como sigue: en 107 actos se atenúa una vez (54,6\%); en 56 actos se atenúa dos veces (28,6\%); en 29 casos se atenúa entre 3 y 7 veces $(14,7 \%)$ y en solo un caso se atenúa 12 veces $(1 \%)$.

El factor nivel de instrucción también presenta variabilidad. De los 291 actos de habla atenuados por los hablantes con estudios primarios, en 137 actos se atenúa solo una vez y en 73 actos se atenúa dos veces, lo que conjuntamente representa el 72,2\% del total de casos atenuados por este grupo de hablantes. El 27,8\% restante se distribuye entre los 3 y los 12 recursos de atenuación por acto de habla, aunque solo en una ocasión se atenúa 12 veces. En segundo orden, de los 259 actos atenuados por los hablantes con estudios secundarios, en 85 ocasiones se atenúa una vez y en 71 oportunidades se atenúa dos veces, lo que constituye el 60,2\% del total de casos atenuados entre una y dos veces por los hablantes del segundo nivel de instrucción. El 39,8\% faltante conforma actos de habla que se atenúan entre 3 y 15 veces; cuando se trata de actos que se atenúan de 10 veces hacia arriba, se trata solo de 6 casos. Luego, de los 200 actos de habla atenuados por informantes con estudios superiores, se observa que en 156 actos se atenúa entre una y dos veces ( $78 \%$ ) y que el $22 \%$ de los casos restantes corresponde a un rango que va entre los 3 y los 10 recursos de atenuación por acto de habla.

En lo que dice relación con la frecuencia de empleo de recursos de atenuación considerando las variables sociales, los datos quedan sintetizados en las Tablas 4, 5 y 6 . 68 | Alpha № 52 (Julio 2021) PÁGS. 53-76. ISSN 07 16-4254 
Tabla 4. Recursos de atenuación y sexo

\begin{tabular}{|l|c|c|c|c|}
\hline \multirow{2}{*}{\multicolumn{1}{|c|}{ Recurso }} & \multicolumn{2}{c|}{ Mujeres } & \multicolumn{2}{c|}{ Hombres } \\
\cline { 2 - 5 } & Frecuencia & \% & Frecuencia & \% \\
\hline Justificaciones & 161 & 53 & 143 & 47 \\
\hline Marcadores discursivos correctores & 125 & 48,8 & 131 & 51,2 \\
\hline $\begin{array}{l}\text { Recursos de expresión de duda } \\
\text { mediante verbos o adverbios }\end{array}$ & 56 & 34,4 & 107 & 65,6 \\
\hline $\begin{array}{l}\text { Recursos de expresión de duda } \\
\text { mediante indicación de incertidumbre }\end{array}$ & 90 & 60,8 & 58 & 39,2 \\
\hline Movimientos concesivos & 76 & 52,8 & 68 & 47,2 \\
\hline $\begin{array}{l}\text { Impersonalizaciones que apelan al } \\
\text { juicio de la mayoría }\end{array}$ & 61 & 47,3 & 68 & 52,7 \\
\hline $\begin{array}{l}\text { Recursos de modificación } \\
\text { morfologica externa }\end{array}$ & 45 & 35,2 & 83 & 64,8 \\
\hline $\begin{array}{l}\text { Marcadores discursivos de } \\
\text { consecución lógica }\end{array}$ & 57 & 46,4 & 66 & 53,6 \\
\hline
\end{tabular}

Como se observa en la tabla precedente, entre los recursos de atenuación de mayor frecuencia, suelen ser los hombres los que presentan un uso más elevado. Se trata en cualquier caso de diferencias mínimas. Sí es relevante, por ejemplo, que en el caso de los recursos de expresión de duda mediante verbos o adverbios y en los recursos de modificación morfológica interna, los hombres duplican a las mujeres en su empleo, mientras que ellas los duplican en el uso de recursos de expresión de duda mediante indicación de incertidumbre.

El resto de los recursos atenuadores, por presentar frecuencias de empleo bajo los 80 casos, no resultan tan relevantes, aunque es necesario destacar, entre otras cosas, que cuando se trata de acotar la opinión, los hombres superan a las mujeres en su empleo; se trata de 43 casos $(56,6 \%)$ versus $33(43,4 \%)$, mientras que cuando se utilizan recursos relacionados con el paralenguaje, sobre todo, risas, las mujeres presentan frecuencias de uso más altas: 41 casos $(59,4 \%)$ versus $22(40,6 \%)$. En todas las categorías se aprecia que no hay grandes diferencias de uso de recursos atenuadores entre hombres y mujeres, lo que se explicaría porque en el total de casos los hombres emplean 877 atenuantes y las mujeres 854 , es decir, hay apenas 23 atenuantes de diferencia entre ambos sexos. 
Tabla 5. Recursos de atenuación y edad

\begin{tabular}{|l|c|c|c|c|c|c|}
\hline \multicolumn{1}{|c|}{ Recurso } & \multicolumn{2}{|c|}{$\mathbf{2 0 - 3 4}$ años } & \multicolumn{2}{c|}{ 35-54 años } & \multicolumn{2}{c|}{$\mathbf{5 5}+$ años } \\
\hline aneci & Frecuenciancia & Frecuencia & \% & Frecuencia & \% \\
\hline Justificaciones & 147 & 48,4 & 91 & 29,9 & 66 & 21,7 \\
\hline $\begin{array}{l}\text { Marcadores } \\
\text { discursivos } \\
\text { correctores }\end{array}$ & 113 & 44,1 & 99 & 38,7 & 44 & 17,1 \\
\hline $\begin{array}{l}\text { Recursos de } \\
\text { expresión de duda } \\
\text { mediante verbos o } \\
\text { adverbios }\end{array}$ & 63 & 42,1 & 81 & 49,5 & 19 & 11,6 \\
\hline $\begin{array}{l}\text { Recursos de } \\
\text { expresión de duda } \\
\text { mediante indicación } \\
\text { de incertidumbre }\end{array}$ & 71 & 43,5 & 81 & 49,7 & 27 & 18,2 \\
\hline $\begin{array}{l}\text { Movimientos } \\
\text { concesivos }\end{array}$ & 80 & 55,5 & 53 & 36,8 & 11 & 7,6 \\
\hline $\begin{array}{l}\text { Impersonalizaciones } \\
\text { que apelan al juicio de } \\
\text { la mayoría }\end{array}$ & 36 & 27,9 & 58 & 44,9 & 35 & 21,7 \\
\hline $\begin{array}{l}\text { Recursos de } \\
\text { modificación } \\
\text { morfológica externa }\end{array}$ & 56 & 43,8 & 55 & 43 & 17 & 13,3 \\
\hline $\begin{array}{l}\text { Marcadores } \\
\text { discursivos de } \\
\text { consecución lógica }\end{array}$ & 42 & 34,1 & 38 & 31 & 43 & 29,8 \\
\hline
\end{tabular}

Acerca de los resultados generales, respecto de la edad y el uso específico de recursos de atenuación, son destacables los casos que implican expresión de duda y las impersonalizaciones, pues difieren mínimamente del patrón general de comportamiento; en estos casos, es el grupo de edad intermedia el que más recursos de atenuación presenta. También es relevante que, aunque se emplea solo en 63 casos, el paralenguaje con función atenuadora se usa en 35 ocasiones por el grupo de hablantes jóvenes, lo que equivale al 55,5\%; en 18 oportunidades por el grupo de 35 a 54 años $(28,6 \%)$; y en 10 ocasiones cuando se trata de hablantes de 55 años y más $(25,9 \%)$. 
Tabla 6. Recursos de atenuación y nivel de instrucción

\begin{tabular}{|l|c|c|c|c|c|c|}
\hline \multirow{2}{*}{ Recurso } & \multicolumn{2}{|c|}{ Básico } & \multicolumn{2}{c|}{ Medio } & \multicolumn{2}{c|}{ Superior } \\
\cline { 2 - 7 } & Frecuencia & \% & Frecuencia & \% & Frecuencia & \% \\
\hline Justificaciones & 148 & 48,8 & 129 & 42,4 & 27 & 8,8 \\
\hline $\begin{array}{l}\text { Marcadores } \\
\text { discursivos } \\
\text { correctores }\end{array}$ & 88 & 34,3 & 117 & 45,7 & 51 & 20 \\
\hline $\begin{array}{l}\text { Recursos de } \\
\text { expresión de duda } \\
\text { mediante verbos o } \\
\text { adverbios }\end{array}$ & 55 & 36,9 & 40 & 27 & 53 & 36,1 \\
\hline $\begin{array}{l}\text { Recursos de } \\
\text { expresión de duda } \\
\text { mediante indicación } \\
\text { de incertidumbre }\end{array}$ & 36 & 22 & 48 & 29,4 & 79 & 48,6 \\
\hline $\begin{array}{l}\text { Movimientos } \\
\text { concesivos }\end{array}$ & 38 & 26,4 & 82 & 56,9 & 24 & 16,7 \\
\hline $\begin{array}{l}\text { Impersonalizaciones } \\
\text { que apelan al juicio } \\
\text { de la mayoría }\end{array}$ & 42 & 32,5 & 53 & 41 & 34 & 26,5 \\
\hline $\begin{array}{l}\text { Recursos de } \\
\text { modificación } \\
\text { morfológica externa }\end{array}$ & 56 & 43,7 & 35 & 27,1 & 37 & 29,2 \\
\hline $\begin{array}{l}\text { Marcadores } \\
\text { discursivos de } \\
\text { consecución lógica }\end{array}$ & 32 & 26 & 68 & 55,2 & 23 & 18,8 \\
\hline
\end{tabular}

Como se señaló con anterioridad, son los hablantes con instrucción superior quienes menos atenúan (397 casos, 22,9\%). En este sentido, llama la atención que, de la misma manera que ocurrió con el factor edad, es el empleo de recursos atenuantes destinados a expresar duda, sea mediante recursos como verbos o adverbios o mediante formas que indiquen incertidumbre o ignorancia, el que difiere del patrón general de comportamiento. En estos casos se observa que los hablantes con estudios superiores superan o se acercan mucho a la frecuencia de empleo que registran los hablantes con estudios básicos. 


\subsubsection{FUNCIONES DE LA ATENUACIÓN Y FACTORES SOCIALES}

Por último, se presentan los resultados de la relación entre las funciones de la atenuación por acto de habla según los factores sexo, edad y nivel de instrucción de los hablantes. Los datos están sacados del total de actos de habla atenuados (750) por factor social.

Tabla 7. Funciones de la atenuación en actos de habla en el corpus PRESEEASantiago de Chile: sexo, edad y nivel de instrucción

\begin{tabular}{|l|r|c|c|c|}
\hline \multicolumn{2}{|c|}{ Factores sociales } & \multicolumn{3}{c|}{ Funciones } \\
\cline { 2 - 5 } \multicolumn{2}{|c|}{} & Autoprotección & Prevención & Reparación \\
\hline \multirow{3}{*}{ Sexo } & Hombre & 326 & 42 & 1 \\
\cline { 2 - 5 } & Mujer & 322 & 54 & 5 \\
\hline \multirow{3}{*}{ Edad } & $20-34$ & 268 & 19 & 1 \\
\cline { 2 - 5 } & $35-54$ & 223 & 42 & 1 \\
\cline { 2 - 5 } & $55+$ años & 155 & 35 & 3 \\
\hline \multirow{3}{*}{$\begin{array}{l}\text { Nivel de } \\
\text { instrucción }\end{array}$} & Bajo & 256 & 52 & 3 \\
\cline { 2 - 5 } & Medio & 293 & 121 & 3 \\
\cline { 2 - 5 } & Alto & 169 & 11 & 0 \\
\hline
\end{tabular}

Como queda especificado en la Tabla 7, en el caso del factor sexo, solo se observan diferencias evidentes en la función de reparación (12 casos de actos de habla atenuados de diferencia). En cuanto al factor edad, se registran diferencias en el uso de atenuantes destinados a autoproteger al hablante, en especial, entre los hablantes de 20 a 34 años y los de 55 años y más. También en relación con la edad, se observa un patrón inverso, donde los hablantes de menos edad son quienes menos actos de habla atenúan. Por último, en lo que se refiere al nivel de instrucción es interesante que la mayor parte de los casos de atenuación se concentre en los hablantes con estudios medios.

\section{CONCLUSIONES}

Las principales conclusiones a las que llega este estudio son las que siguen:

1. En las nueve horas de grabación se registran 750/1901 (39,5\%) actos de habla atenuados. Más específicamente, se concluyó que suelen utilizarse entre uno y dos recursos de atenuación por cada acto de habla $(68,9 \%)$, y que se atenúa más el modus, de manera que se reduce la fuerza ilocutiva del acto de habla. Estos resultados son similares a los expuestos en los estudios acerca de otras variedades del español con materiales del PRESEEA. 
2. En relación con el análisis de los factores lingüísticos, se puede concluir que en el corpus PRESEEA de Santiago de Chile se emplean 21 de las 25 categorías propuestas en Cestero y Albelda (2012). Los atenuadores más utilizados son las justificaciones y excusas. Le siguen, en orden de frecuencia, los marcadores del discurso correctores, la expresión de aserciones en forma de duda o de probabilidad mediante el empleo de verbos o adverbios, la expresión de aserciones en forma de duda o de probabilidad mediante indicación de incertidumbre o incompetencia, los movimientos concesivos, las impersonalizaciones que apelan al juicio de la mayoría o a un interlocutor general, los recursos de modificación morfológica externa y los marcadores discursivos de consecución lógica.

3. En la dimensión pragmático-discursiva se pudo constatar que los atenuadores suelen tener la función de autoproteger la imagen de los informantes.

4. En lo que respecta al estudio de los factores sociales, las principales conclusiones indican que tanto en los actos de habla atenuados como en el uso específico de recursos atenuantes, en el factor sexo las frecuencias de empleo son prácticamente idénticas; en cuanto al factor edad, se vio que el empleo de recursos atenuadores disminuye conforme aumenta la edad. Finalmente, en el caso del factor nivel de instrucción se concluye que en el grupo de hablantes con estudios superiores, aparecen menos actos de habla atenuados y menos recursos en dichos actos de habla. La mayor variabilidad se produce en las mujeres, porque las de estudios universitarios -sobre todo las de 55 años y más- emplean muy pocos recursos atenuadores en comparación al resto de los hablantes de la muestra. Se comprueba, además, que los hablantes jóvenes son los que más atenúan y aquellos con estudios universitarios los que menos atenúan.

Finalmente, se debe señalar que los datos descriptivos aportados, así como la presentación de hallazgos generales respecto de la convergencia y la divergencia con otras variedades del español, corroboran la relevancia del macrocorpus PRESEEA. A este respeto, se deben tener en consideración los trabajos de Cestero (2020) y Albelda et al. (2020). No obstante, hay que considerar que se trata de conclusiones que se aplican al género investigado, pero que eventualmente podrían diferir de aquellas a las que se podría llegar en el estudio de la conversación espontánea, sobre todo, por el carácter +/- coloquial y + -- formal de la situación comunicativa.

Este trabajo se enmarca en el proyecto de investigación "La atenuación lingüística en el español chileno: enfoque pragmalingüístico y sociopragmático" (ANID/CONICYT Fondecyt Regular 1200003). 
Silvana Guerrero González

\section{OBRAS CITADAS}

Albelda, Marta (2018). "Variación sociolingüística de los mecanismos mitigadores: diferencias de uso en edad y sexo". Cultura, lenguaje y representación volumen XIX, 7-29.

(2016). "Sobre la incidencia de la imagen en la atenuación pragmática". Revista Internacional de Lingüística Iberoamericana 27 (1), 19-32.

(2013). La atenuación: tipos y estrategias. En Gómez Molina, José Ramón (coord.), El español de Valencia. Estudio sociolingüístico. Frankfurt am Main: Peter Lang, 315-343.

(2012). Variación sociolingüística en las estrategias de atenuación del corpus PRESEEA-VALENCIA del sociolecto alto. En Cestero, Ana, Isabel Molina y Florentino Paredes (eds.), La lengua, lugar de encuentro. Actas del XVI Congreso Internacional de la Alfal. Alcalá de Henares: Servicio de Publicaciones de la Universidad de Alcalá, 1857-1866.

(2011). Estudio sociolingüístico (piloto) de la atenuación en el corpus PRESEEA de Valencia. En Actas del IX Congreso Internacional de Lingüística General. Valladolid: Universidad de Valladolid, 35-55.

Albelda, Marta; Briz, Antonio; Cestero, Ana María; Kotwica, Dorota y Villalba, Cristina (2014). "Ficha metodológica para el análisis pragmático de la atenuación en corpus discursivos del español (ES.POR.ATENUACIÓN)". Oralia 17, 7-62.

Albelda, Marta y Cestero, Ana María (2011). "De nuevo, sobre los procedimientos de atenuación lingüística”. Español Actual 96, 121-155.

Albelda, Marta; Cestero, Ana María; Guerrero, Silvana y Samper, Marta (2020). Variación sociopragmática y geolectal en el uso de la atenuación. Lengua y Habla 24, 1-53.

Albelda, Marta y Nihatsch, Wiltrud (2017). Atenuación e intensificación en géneros discursivos. Madrid/Frankfurt: Iberoamericana/Vervuert.

Briz, Antonio (2012). La (no)atenuación y la (des)cortesía, lo linguístico y lo social: ¿son pareja? En Julio Escamilla et alii (eds.), Miradas multidisciplinares a los fenómenos de cortesía y descortesía en el mundo hispánico. Barranquilla: Universidad del Atlántico-Programa EDICE, 33-75.

— (2007). "Para un análisis semántico, pragmático y sociopragmático de la cortesía atenuadora en España y América", Lingüística Española Actual 29/1, 5-40.

(1995). La atenuación en la conversación coloquial. Una categoría pragmática. En Cortés, Luis. (ed.) El español coloquial: actas del I Simposio sobre análisis del discurso oral. Almería: Universidad de Almería, 103-122. 
Briz, Antonio y Albelda, Marta (2013). "Una propuesta teórica y metodológica para el análisis de la atenuación lingüística en español y portugués. La base de un proyecto en común (ES.POR.ATENUACIÓN)". Onomázein 27 (2), 288-319.

Briz, Antonio y Estellés, María (2010). On the relationship between Attenuation, Discourse Particles and Position”. En Kaltenböck, Gunther, Wiltrud Mihatsch y Stefan Schneider (eds.), Studies in Pragmatics 9. New Approaches to Hedging. United Kingdon: Emerald Group Publishing, 289-304.

Cestero, Ana María (2020). "Uses and resources of mitigation, in cotrast". Spanish in Context, 17.2.

(2017). 'La atenuación en el habla de Madrid: patrones sociopragmáticos". RILCE $33(1), 57-86$.

(2015). La atenuación lingüística en el habla de Madrid: un fenómeno sociopragmático variable. En Cestero, Ana M., Molina, Isabel y Florentino Paredes (eds.), Patrones sociolinguísticos de Madrid. Bern: Peter Lang, 365-412.

— (2012a). Recursos lingüísticos de atenuación en el habla de Madrid estudio sociopragmático. En Jiménez Juliá, Tomás, Belén López Meirama, Victoria Vázquez Rozas, Alexandre Veiga Rodríguez (coord.), Cum corde et in nova grammatica: estudios ofrecidos a Guillermo Rojo. Universidad de Santiago de Compostela, 233-246.

(2012b). Estudio sociolingüístico de la atenuación en el corpus PRESEEA-Madrid. En Cestero, Ana, Isabel Molina y Florentino Paredes (eds.), La lengua, lugar de encuentro. Actas del XVI Congreso Internacional de la Alfal, Alcalá de Henares: Servicio de Publicaciones de la Universidad de Alcalá, 1897-1906.

— (2011). Las estrategias de atenuación: estudio sociolingüístico. En Actas del IX Congreso Internacional de Lingüística General. Valladolid: Universidad de Valladolid, 525-542.

Cestero, Ana María y Lidia Rodríguez Alfano (2014). “Análisis de la atenuación. Guía de estudio de la atenuación”. Versión Julio-2008. Recuperado en noviembre de 2019 de: http://preseea.linguas.net

Cestero, Ana y Albelda, Marta (2012). "La atenuación lingüística como fenómeno variable". Oralia 15, 77-124.

González Riffo, Javier (2017). Estrategias de atenuación en narraciones de experiencia personal de hablantes de Santiago de Chile: un estudio sociopragmático. Tesis para optar al grado de Magíster en Lingüística. Santiago: Universidad de Chile.

González Riffo, Javier y Guerrero González, Silvana (2018). “Recursos de atenuación en la 'orientación' de narrativas personales orales desde una perspectiva sociopragmática". Nueva revista del Pacífico 68, 62-81.

(2017). "Estrategias de atenuación en narraciones conversacionales". Lengua y Habla 21, 29-44. 
- (2016). "Estudio sociolingüístico del empleo de gradación de valoraciones inscritas en discursos argumentativos en el corpus PRESEEA de Santiago de Chile". Linred, 14. Recuperado en enero de 2019 de: http://www.linred.es/articulos_pdf/LR-articulo-10102016.pdf

González, Carlos y Hugo, Evelyn (2012). "Cuando te lo piden, uno no siempre sabe que decir". Uno y tú como estrategias evidenciales en el español de Chile”. Actas del IV Congreso Internacional de Letras 2010, "Transformaciones culturales. Debates de la teoría, la crítica y la lingüística en el Bicentenario”, Universidad de Buenos Aires.

Molina, Isabel (2015). "Estrategias de atenuación en el barrio de Salamanca de Madrid". En Cestero, Ana M., Molina, Isabel y Forentino Paredes (eds.), Patrones sociolingüísticos de Madrid. Bern: Peter Lang, 349-364.

(2005). "Estrategias de atenuación en el barrio de Salamanca de Madrid". Presentación en XIV Congreso Internacional ALFAL. Memorias, vol. I, sección 'Dialectología y Sociolingüística'. Monterrey, Universidad Autónoma de Nuevo León.

Puga, Juana (1997). La atenuación en el castellano de Chile. Un enfoque pragmalingüístico. Valencia: Universidad de Valencia.

Rodríguez Alfano, Lidia (2018). "La atenuación en justificaciones argumentativas en el corpus Monterrey-PRESEEA”. Anuario de Letras. Lingüística y Filología 6, 219250.

Samper, Marta (2018). "Un cambio en tiempo real: la atenuación entre hablantes universitarios de Las Palmas de Gran Canaria". RILCE 34 (3), 1259-1279.

(2017). Análisis sociolinguístico de la atenuación en el español de Las Palmas de Gran Canaria. En Albelda, Marta y Wiltrud Nihatsch. (eds.). Atenuación e intensificación en géneros discursivos. Madrid/Frankfurt: Iberoamericana/Vervuert.

(2013). "La atenuación lingüística en el español de Las Palmas de Gran Canaria”. En LEA 35(2), 181-204.

Torres, Yasmín y Rodríguez, Yolanda (2016). "La atenuación en Barranquilla: estudio sociopragmático". Cuadernos de Lingüística Hispánica 30, 55-79. 